\title{
Editorial
}

\section{Cardiac biomarkers for outcome prediction in infant bronchiolitis: Too soon to discard troponin?}

\section{A R T I C L E I N F O}

\section{Keywords}

Cardiac troponins

NT-proBNP

High-sensitivity methods

Myocardial injury

Pediatric cardiology

Reference intervals

\begin{abstract}
A B S T R A C T
Background: Acute bronchiolitis, usually caused by the respiratory syncytial virus, is the most common cause of severe respiratory distress in infants. The clinical relevance of cardiac biomarkers for diagnosis and prognosis of bronchiolitis in infants is still controversial.

Aim: This Editorial discuss the results of a recent on the clinical relevance of cardiac specific biomarkers, NTproBNP and cardiac troponin I (cTnI) in 40 infants with bronchiolitis, 37\% requiring admission in the ICU.

Results: NT-proBNP levels were significantly associated with prolonged hospitalization and duration of oxygen therapy, while cTnI levels, measured with high-sensitivity (hs) methods, did not. Furthermore NT-proBNP was significantly correlated with left and right ventricular functional echocardiographic parameters, while hs-cTnI was not associated with any echocardiographic parameter.

Discussion: Recent results confirm that NT-proBNP assay has a crucial role in the diagnosis, prognosis and followup of patients with cardiac disease not only in adult, but even in pediatric age). On the other hand, the clinical usefulness of assay of hs-cTnI and hs-cTnT in pediatric cardiology, at present time, may be greatly limited by the lack of reliable reference intervals.

Conclusions: Further well-designed multi-center studies are needed to more accurately evaluate the clinical relevance of cardio-specific biomarkers, and in particular of hs-cTnI and hs-cTnT assay, on prognosis of infants with bronchiolitis.
\end{abstract}

Acute bronchiolitis, usually caused by the respiratory syncytial virus, is the most common cause of severe respiratory distress in infants $[1,2]$. In high-income countries, approximately one out of eight infants hospitalized with bronchiolitis requires admission to Intensive Care Unit (ICU) for respiratory support, and acute bronchiolitis represents the most common reason for hospital admission during the first year of life $[1,2]$. Even if bronchiolitis is a primary lung disorder, a recent metaanalysis reported that some degree of cardiovascular involvement is not infrequent [3]. Myocardial dysfunction (pooled incidence of 5\%, CI 95\%: $1-9 \%$ ) and/or pulmonary hypertension (pooled incidence of $20 \%$, CI 95\%: $11-30 \%$ ) may be present even in early disease stages and are significantly related to clinical severity and adverse outcome [3]. Furthermore, a recent meta-analysis showed that NT-proBNP, but not cardiac troponins (cTn), accurately and independently predicted the presence of myocardial impairment by echocardiography [3]. However, these results [3] were based on data reported by just 5 studies, including a relatively limited sample size (e.g., 272 children with acute bronchiolitis). In addition, only one study investigated both NT-proBNP and cardiac troponin I (cTnI), but the echocardiographic assessment did not include strain evaluation [3].

In this issue of Clinica Chimica Acta, Castellano-Martinez et al. [4], starting from the results of the meta-analysis by the same investigators [3], evaluated in a single-center study the association between NTproBNP and high-sensitivity (hs) cTnI and altered echocardiographic findings, disease severity and outcomes in 40 infants with bronchiolitis, $37 \%$ requiring admission in the ICU [4]. Exclusion criteria were: known risk factors for severe bronchiolitis, presence of chronic diseases, acquired or congenital immunodeficiency, prematurity (less than 36 weeks of gestational age), initiation of respiratory support (invasive or non-invasive) or administration of epinephrine or intravenous fluid before enrollment, incomplete intervention or medical records, and poor-quality echocardiographic images. Median admission NT-proBNP and hs-cTnI were $1176 \mathrm{ng} / \mathrm{L}$ (interquartile range 520-3030 ng/L) and 12 (5-21 ng/L), respectively [4]. Increased values of NT-proBNP (cut-off $1121 \mathrm{ng} / \mathrm{L}$ ) and hs-cTnI (cut-off $26.2 \mathrm{ng} / \mathrm{L}$ ) were observed in 20 (50\%) and $8(20 \%)$ cases, respectively. Moreover, NT-proBNP and hs-cTnI values showed a significant positive correlation $(U=0.45 ; p=0.003$, Spearman' rank correlation test).

Considering the logistic regression analyses, adjusted for age, weight and gender, infants with NT-proBNP levels $>1121 \mathrm{ng} / \mathrm{L}$ were at higher risk for ICU admission (odds ratio [OR] 9.5, 95\% confidence interval [CI]: $1.4-64, p=0.020$ ) [4]. Considering the adjusted multivariate regression analysis, NT-proBNP were significantly associated with prolonged hospitalization $(\mathrm{p}=0.012)$ and duration of oxygen therapy $(\mathrm{p}=$ 0.004 ) [4]. Admission NT-proBNP had an area under curve (AUC) of 0.801 to predict ICU admission (95\% CI: 0.691-0.902), with the best cut-off point of $836 \mathrm{ng} / \mathrm{L}$, corresponding to a sensitivity of $93 \%$, a specificity of $56 \%$, a positive predictive value of $56 \%$, and a negative 
predictive value of 93\% [4]. Furthermore, the Authors did not find significant differences in hs-cTnI among patients who required admission to ICU and those who did not $(\mathrm{p}=0.866)$. Moreover, increased hscTnI ( $>26.2 \mathrm{ng} / \mathrm{L}$ ) were associated with oxygen administration duration ( $p=0.017)$, but this association did not remain significant after adjusting for age, weight and gender. Finally, there were no significant correlations between hs-cTnI levels and length of ICU stay and duration of hospital stay [4]. NT-proBNP was significantly correlated ( $p<0.001$ ) with left and right ventricular functional echocardiographic parameters, while hs-cTnI was not associated with any echocardiographic parameter [4]. The Authors concluded that early elevation of NT-proBNP, but not hs-cTnI, could be used as marker of disease severity in bronchiolitis [4].

This study [4] certainly reports some interesting and original data. However, some methodological issues deserve further consideration. As acknowledged by the Authors, this study [4] has some limitations, particularly the small sample size and the lack of a control group. The small sample size, in particular the low number of infants with severe disease and increased levels of both cardiospecific biomarkers (only 8 cases), is a critical issue for logistic and multivariate regression analyses evaluating the relation of biomarkers to outcome. Moreover, the lack of a control group further complicates the interpretation of results regarding cardiac biomarkers. Indeed, some data exist on the reference interval of cardiac natriuretic peptides (including NT-proBNP) in healthy infants [5-7]. Conversely, only sparse and limited data are available on reference intervals for hs-cTnI and hs-cTnT in the pediatric age $[8,9]$. In addition, there are several commercial assays for hs-TnI, which differ widely in terms of reference intervals and cut-off values in adult healthy subjects $[10,11]$. The cut-offs considered for cardiac biomarkers by Castellano-Martinez et al. [4], especially those for hs-cTnI (i.e, $26.2 \mathrm{ng} / \mathrm{L}$ ), which are extrapolated from data reported by the manufacturer for adult reference population, may be inaccurate, and so the estimated frequency of myocardial injury in infants with bronchiolitis may be mistaken.

The combined measure of cardiac specific biomarkers is a valuable resource for clinicians because these biomarkers add independent and additional pathophysiological and clinical information in several cardiac and extra-cardiac conditions [12]. In adults with sepsis, NT-proBNP elevation is driven by hypoxemia, activation of pro-inflammatory cytokines, increase in right atrial and ventricular pressure, tachycardia (or even supraventricular arrythmias), and in the most severe case ventricular dysfunction [12]. It is conceivable that the same pathophysiological mechanisms are responsible for increased NT-proBNP in infant with bronchiolitis. Myocardial injury is present when blood levels of hscTnI or hs-cTnT are increased above the upper reference limit, indicating a necrosis of cardiomyocytes with the release of biomarker into circulation [13]. In the study by Castellano-Martinez et al. [4], all the 8 infants with increased hs-cTnI have also increased NT-proBNP levels. Therefore, the combined increase in NT-proBNP and hs-cTnI levels indicates that both cardiac dysfunction and myocardial injury are present in these infants with bronchiolitis. These results support the accumulating evidence that both pediatric and adult patients with cardiac disease usually have a more severe prognosis when both cardio-specific biomarkers are increased [12]. As also suggested by Castellano-Martinez et al. [4], the necrosis of cardiomyocytes, inducing cTnI release, usually occurs after prolonged and intense hypoxemia or myocardial ischemia, which may be rare in infants with bronchiolitis. Similar results have been reported in patients (both adults and infants) with SARS-CoV-2. In these patients, myocardial injury is common and strongly associated with severe prognosis [14]. In particular, patients with SARS-CoV-2 and myocardial injury have worse hypoxemia and outcome [14].

As for the comparison between laboratory and imaging results, it is important to note that the measurement of cardiac troponins using hscTn assays is able to detect the injury of about $10-40 \mathrm{mg}$ of myocardium, which is too small to detect by non-invasive imaging techniques [15-17]. Therefore, a low degree of association between hs-cTnI assay and echocardiographic results reported in the study by Castellano-
Martinez et al. [4] may be expected, especially taking into consideration the enrolled population, including a relatively small number of patients with myocardial injury $(\mathrm{n}=8)$.

The results reported by Castellano-Martinez et al. in their metaanalysis [3] and clinical study [4] confirm that the assay of cardiac natriuretic peptides (in particular NT-proBNP) plays a crucial role in the diagnosis, prognosis and follow-up of patients with cardiac disease not only in adult $[12,14]$, but even in pediatric age $[5-8,14]$ On the other hand, the clinical usefulness of assay of hs-cTnI and hs-cTnT in pediatric cardiology, at present time, may be greatly limited by the lack of reliable reference intervals.

Due to their excellent analytical performance and unique pathophysiological characteristics (especially their very low intra-individual variation and the strict relation to physiological myocardial renewal) [15-17], the measurement of hs-cTnI and hs-cTnT should achieve a fundamental role also in pediatric age (including neonates, infants, children and adolescents), allowing to better understand both the physiological development and adaption to physical exercise of cardiovascular system, and also the mechanisms and clinical features of cardiac disease. We believe that the minor role compared to other biomarkers (in particular cardiac natriuretic peptides), now played by cardiac troponin assay in pediatric cardiology, is mainly due to the lack of reliable reference intervals, divided according to age (from neonatal period to adolescence), and also to sex for boys and girls. Unfortunately, the reliable identification of 99th percentile values according to the international guidelines $[13,18]$, it is a very difficult task $[8,10,17]$, which would require an extraordinary effort and possibly the involvement and support of National Scientific Societies of Pediatric Cardiology and Laboratory Medicines.

In conclusions, further well-designed multi-center studies are needed to more accurately evaluate the clinical relevance of cardio-specific biomarkers, and in particular of hs-cTnI and hs-cTnT assay, on prognosis of infants with bronchiolitis.

\section{Declaration of Competing Interest}

The authors declare that they have no known competing financial interests or personal relationships that could have appeared to influence the work reported in this paper.

\section{References}

[1] A.S. Florin, A.C. Plint, J.J. Zorc, Viral bronchliolitis, Lancet 389 (2017) 211-224, https://doi.org/10.1016/S0140-6736(16)30951-5.

[2] L.J. Schlapbach, L. Straney, B. Gelbart, J. Alexander, D. Franklin, J. Beca, J. A. Whitty, S. Ganu, B. Wilkins, A. Slater, E. Croston, S. Erikson, A. Schilbler, Burden of disease and change in practice in critically ill infants with bronchiolitis, Eur. Respir. J. 49 (2017) 1601648, https://doi.org/10.1183/13993003.01648-2016.

[3] M. Rodriguez-Gonzalez, A.A. Perez-Reviriego, A. Castellano-Martinez, H. M. Cascales-Poyatos, The Assessment of myocardial strain by cardiac imaging in healthy infants with acute bronchiolitis: A systematic review and metaanalysis, Diagnostics (Basel) 10 (6) (2020), https://doi.org/10.3390/diagnostics10060382.

[4] A. Castelano-Martine, et al., Early elevated NT-proBNP but not Troponin I is associated with severe bronchiolitis in infants, Clin. Chim. Acta (2021) in press (CCACTA-D-21-00470R1).

[5] T.S. Mir, M. Flato, J. Falkenberg, M. Haddad, R. Budden, J. Weil, Plasma concentrations of $\mathrm{N}$-terminal brain natriuretic peptide in healthy children, adolescents, and young adults: effect of age and gender, Pediatr. Cardiol. 27 (2006) 73-77, https://doi.org/10.1007/s00246-005-1022-4.

[6] A. Nir, A. Lindinger, M. Rauh, B. Bar-Oz, S. Lear, L. Schwachtgen, A. Koch, T.S. Mir, NT-Pro-B-type natriuretic peptide in infants and children: reference values based on combined data from four studies, Pediatr. Cardiol. 30 (3-8) (2009), https://doi. org/10.1007/s00246-008-9258-4.

[7] M. Cantinotti, Y. Law, S. Vittorini, M. Crocetti, M-. Marotta, B. Murzi, A. Clerico, The potential and limitations of plasma BNP measurement in the diagnosis, prognosis, and management of children with heart failure due to congenital cardiac disease: an update, Heart Fail. Rev. 19 (2014) 727-742, https://doi.org/10.1007/ s10741-014-9422-2.

[8] C. Caselli, G. Cangemi, S. Masotti, Plasma cardiac troponin I concentrations in healthy neonates, children and adolescents measured with a high sensitive immunoassay method: High sensitive troponin I in pediatric age, Clin. Chim. Acta 458 (2016) 68-71, https://doi.org/10.1016/j.cca.2016.04.029. 
[9] P. Jehlicka, D. Rajdl, E. Sladkova, A. Sykorova, J. Sykora, Dynamic changes of high-sensitivity troponin T concentration during infancy: Clinical implications, Physiol. Res. 70 (2021) 27-32, https://doi.org/10.33549/physiolres.934453.

[10] A. Clerico, M. Zaninotto, A. Ripoli, S. Masotti, C. Prontera, C. Passino, M. Plebani, The 99th percentile of reference population for cTnI and cTnT assay: methodology, pathophysiology and clinical implications, Clin. Chem. Lab. Med. 55 (2017) 1634-1651, https://doi.org/10.1515/cclm-2016-0933.

[11] A. Clerico, A. Ripoli, M. Zaninotto, S. Masotti, V. Musetti, M. Ciaccio, R. Aloe, S. Rizzardi, R. Dittadi, C. Carozza, T. Fasano, M. Perrone, A. de Santis, C. Prontera, D. Riggio, C. Guiotto, M. Migliardi, S. Bernardini, M-. Plebani, Head-to-head comparison of plasma cTnI concentration values measured with three highsensitivity methods in a large Italian population of healthy volunteers and patients admitted to emergency department with acute coronary syndrome: A multi-center study, Clin. Chim. Acta 496 (2019) 25-34, https://doi.org/10.1016/J. cca.2029.06.012.

[12] M.A. Perrone, M. Zaninotto, S. Masotti, V. Musetti, A. Padoan, C. Prontera, M. Plebani, C. Passino, F. Romeo, S. Bernardini, A. Clerico, The combined measurement of high-sensitivity cardiac troponins and natriuretic peptides: a useful tool for clinicians? J. Cardiovasc. Med. 21 (2020) 953-963, https://doi.org/ 10.2459/JCM.0000000000001022.

[13] K. Thygesen, J.S. Alpert, A.S. Jaffe, B.R. Chaitman, J.J. Bax, D.A. Morrow, H D. White, Executive Group on behalf of the Joint European Society of Cardiology (ESC)/American College of Cardiology (ACC)/American Heart Association (AHA)/ World Heart Federation (WHF) Task Force for the Universal Definition of Myocardial Infarction. Fourth Universal Definition of Myocardal Infarction, J. Am. Coll. Cardiol. 72 (2018) 2231-2264, https://doi.org/10.1016/j.jacc. 2018.08.1038.

[14] Y. Sandoval, J.J. Januzzi, A.S. Jaffe, Cardiac troponin for assessment of myocardia injury in COVID-19. JAAC review topic of the week, J. Am. Col. Cardiol. 76 (2020) 1244-1258, https://doi.org/10.1016/j.jacc.2020.06.068.

[15] A. Clerico, A. Giannoni, C. Prontera, S. Giovannini, High-sensitivity troponin: A new tool for pathophysiological investigation and clinical practice, Adv. Clin. Chem. 49 (2009) 1-30, https://doi.org/10.1016/S0065-2423(09)49001-2.
[16] J. Marjot, T.E. Kaier, E.D. Martin, S.S. Reji, O.N. Copeland, M. Iqbal, B. Goodson, S. Hamren, S.E. Harding, M.S. Marber, Quantifying the release of biomarkers of myocardial necrosis from cardiac myocytes and intact myocardium, Clin. Chem. 63 (2017) 990-996, https://doi.org/10.1373/clinchem.2016.264648.

[17] A. Clerico, M. Zaninotto, A. Padoan, S. Masotti, V. Musetti, C. Prontera, R. Ndreu, G. Zucchelli, C. Passino, M. Migliardi, M. Plebani, Evaluation of analytical performance of immunoassay methods for cTnI and cTnT: From theory to practice, Adv. Clin. Chem. 93 (2019) 239-262, https://doi.org/10.1016/bs. acc. 2019.07.005.

[18] A.H.B. Wu, R.H. Christenson, D.N. Greene, A.S. Jaffe, P.A. Kavsak, J. OrdonezLianos, F.S. Apple, Clinical laboratory practice recommendations for the use of cardiac troponin in acute coronary syndrome: Expert opinion from the Academy of the American Association for Clinical Chemistry and the Task Force on Clinical Applications of Cardiac Bio-Markers of the International Federation of, Clinical Chemistry and Laboratory Medicine, Clin. Chem. 64 (2018) 645-655, https://doi.org/10.1373/clinchem.2017.277186.

Aldo Clerico*, Alberto Aimo, Massimiliano Cantinotti Fondazione CNR-Regione Toscana G. Monasterio and Scuola Superiore Sant'Anna, Pisa, Italy

Corresponding author at: Laboratory of Cardiovascular Endocrinology and Cell Biology, Department of Laboratory Medicine, Fondazione CNR

Toscana G. Monasterio, Scuola Superiore Sant'Anna, Via Trieste 41, 56126 Pisa, Italy. E-mail address: clerico@ftgm.it (A. Clerico). 